\title{
Measuring jumps during granular chute-flows using X-ray radiography
}

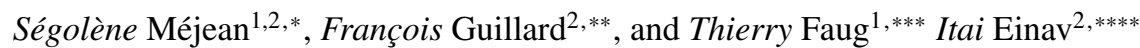 \\ ${ }^{1}$ Univ. Grenoble Alpes, INRAE, UR ETNA, F-38402 St Martin d'Hères, France \\ ${ }^{2}$ School of Civil Engineering, The University of Sydney, Sydney, NSW 2006, Australia
}

\begin{abstract}
The present paper describes laboratory tests on steady granular flows down a smooth chute using $\mathrm{X}$-ray radiography. By using an original granular chute set-up with a gate at its end, standing discontinuites in height, velocity and density, namely jumps, were produced during the granular flows. The X-ray radiography was successful to get consistent measurements of the free-surface and density profiles of the flows along the chute under different flow states: the (nearly) uniform flows before the jumps, the gradually-varied flows after the jumps, and the highly non-uniform flows across the jumps. This demonstrates the efficiency of the X-ray radiography to measure granular flows.
\end{abstract}

\section{Introduction}

Flows of granular materials are ubiquitous in nature and industrial processes. Discontinuities in height, velocity and density, namely jumps, occur during the transition between supercritical and subcritical states of the granular flows. Such flow discontinuities, called jumps by analogy to the canonical case in hydraulics, form in multiple situations. The impact of granular flows on walls for instance produce a granular jump [1] that become a key process for the design of protection dams against landslides and avalanches [3]. Jumps in dense granular currents were recently reported as a key process for the interpretation of deposits lefts by pyroclastic flows in vulcanology [7]. Granular jumps are also observed in industrial applications that imply the transport of particles, for instance during silo discharge problems $[6,8]$ or in pneumatic conveying systems [5]. Understanding the physics of granular flows and predicting the geometry of granular jumps is therefore crucial. In the present paper, standing jumps are produced in free-surface flows of granular materials down a smooth chute and the jumps are measured using the dynamic $\mathrm{X}$ ray radiography developed by [4]. The X-ray technique used gives access to accurate measurements of the freesurface and density profiles of the granular flows along the chute.

The present paper is organized as it follows. Section 2 describes the granular chute set-up used to produce the jumps and gives details about the X-ray radiography. Section 3 present examples of free-surface and density profiles along the chute measured by X-ray radiography, showing how robust and powerful the technique is (i) to give consistent measurements on the density of granular flows and

\footnotetext{
*e-mail: segolene.mejean@gmail.com

**e-mail: francois.guillard@sydney.edu.au

***e-mail: thierry.faug@inrae.fr

****e-mail: itai.einav@sydney.edu.au

A video is available at https://doi.org/10.48448/3j2z-th91
}

(ii) to decipher some key details of the granular jumps. Section 4 concludes the paper by addressing the interest of using X-ray radiography to investigate granular chuteflows and some further developments needed.

\section{Experimental Methods}

\subsection{The granular jump chute-flow set-up}

The granular chute used in the present study to produce standing jumps in flows of dry granular materials was initially developed by [2]. The granular chute is $1 \mathrm{~m}$ long with width $W=10 \mathrm{~cm}$ (see Fig. 1), and equipped with a tank at the top to store grains before release them into the chute. A gate with opening height $H$ at the exit of the tank allows to control the mass discharge $Q_{m}$ which is controlled by a relation in the form of a Berveloo law (see details in [2]): $Q_{m}=C \phi_{\infty} \rho_{P} \sqrt{g H \sin \zeta} H W$, where $\rho_{P}$ is the particle density, $g$ the acceleration of gravity, $\zeta$ the slope angle of the chute, $\phi_{\infty}$ the maximum close packing (considered as equal to 0.64 for monodisperse glass beads) and $C$ a coefficient which mainly depends on grain properties $(C=0.72$ for glass beads of diameter $d=1.2 \mathrm{~mm}$, as used in the experiments here and early calibrated by [2]).

The originality of that granular chute is that it is equipped with another gate which allows to control the mass discharge at the end of the chute (see Fig. 1). At the beginning of one test, that gate is kept close after releasing the grain at the tank exit. When the grains reach the gate at the end of the chute, a jump is formed and propagates upstream of the gate, while grains pile-up between the propagating jump and the downstream gate. At one point, when a sufficient amount of static grains has piledup and before that the jump propagates far upstream and reaches the tank (which would lead to a complete jamming of the grains all along the chute and inside the tank), the gate at the end of the chute is lifted in order to make the 
granular jump stationary. The standing jump formed is stable when the mass discharge at the end of the chute exactly equals the mass discharge at the tank exit (entrance of the chute). This experiment allows to produce standing jumps that are stable over a wide range of slope angle and mass discharge, as earlier reported by [2].
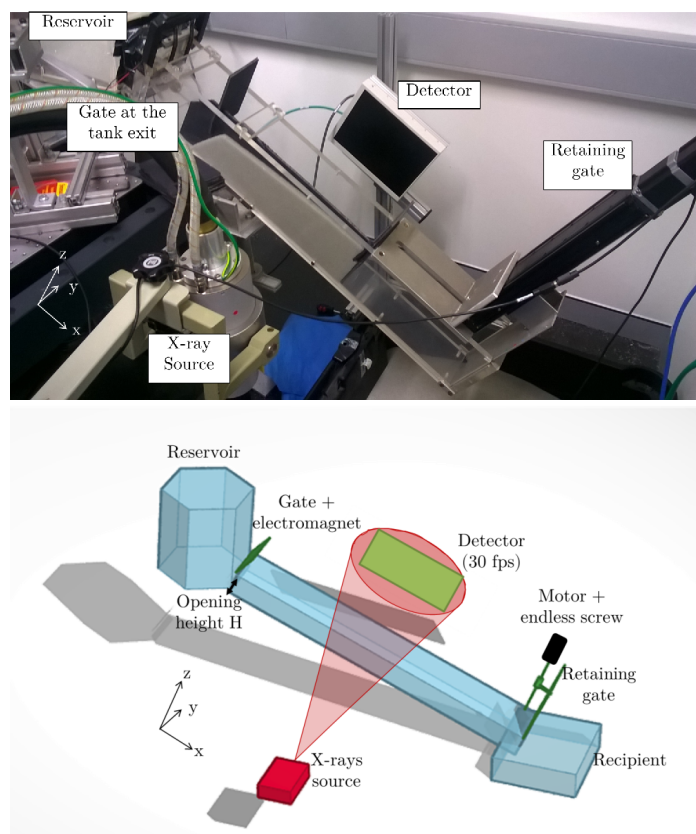

Figure 1. Photograph of the laboratory granular chute to produce standing jumps, installed in the X-ray room (top panel). Sketch of the granular chute showing the main elements: tank, channel, two automatic gates at the tank exit and end of the chute, and $\mathrm{X}$-ray source and detector.

In order to perform X-ray radiography without unecessary human exposure to ionising radiations, the chute had to be instrumented with remote control. An electromagnet was adapted on the input flow gate to control the flow initiation (see Fig. 1). At the end of the chute, a second gate opening was precisely controlled using a translation stage (see Fig. 1). This gate is initially closed to form the jump at the beginning of the experiment, and then opened and ajusted in order to obtain a stationary jump in front of the detector. This positioning of the standing jump was sometimes challenging, in particular when the propagating jump was moving very fast upstream.

\subsection{X-ray radiography of the granular jumps}

The X-ray device is composed of a source emitting Xrays perpendicularly to the area of interest along the chute, where the jump is set stationary, and a detector on the other side of the chute, which measures the intensity of the X-rays after having crossed the flume and the granular medium inside, at a frequency of 30 frames per second (see Fig. 1).

The detector measures the intensity in every pixel (960 pixels along the $x$-axis that follows the channel slope angle, and 768 pixels along the $z$-axis, perpendicular to the $x$-axis), and converts it into a number between 0 and 65535. An example of this raw image at a given time $t$ is shown in Fig. 2 for a jump made of glass beads (top panel) and a jump made of rice grains (bottom panel).

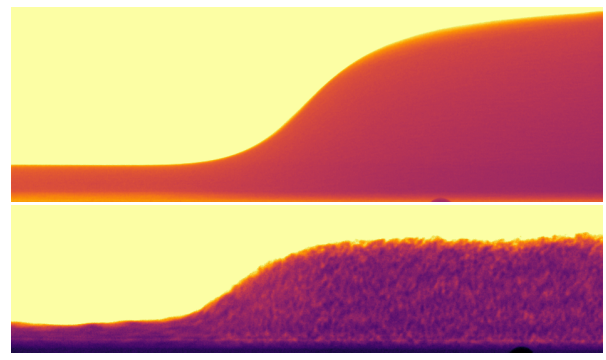

Figure 2. Examples of raw images of the jumps obtained by Xray radiography at a given time $t$ : glass beads for $H=35 \mathrm{~mm}$ and $\zeta=30^{\circ}$ (top panel) and rice grains for $H=35 \mathrm{~mm}$ and $\zeta=35^{\circ}$ (bottom panel). The particles are flowing from left to right.

It can be noted some differences between the spherical grains (glass beads) that produce a smooth flow freesurface and a quite uniform radiogram and the elongated particles (rice grains) that produce a much more heckled free-surface and a much less uniform radiogram. In the latter case, one can detect that the orientation of the grains changes when moving from the incoming flow before the jump towards the flow downstream of the jump: the rice grains are nearly aligned with the chute bottom before the jump, while they tend to align vertically (in a direction normal to the chute bottom) after the jump.

The measured intensity on a given pixel at location $(x, z)$ of the detector is the following (neglecting beam hardening):

$$
I(x, z)=I_{0} \exp \left(\int_{\ell}-\mu_{a t t} \rho_{m}(x, z, \ell) \mathrm{d} \ell\right),
$$

where $I_{0}$ is the intensity of the source, $\ell$ the ray path inside the medium, $\mu_{\text {att }}$ the attenuation coefficient and $\rho_{m}(x, z, \ell)$ the density of the medium crossed by the ray that reaches the detector at point $(x, z)$ averaged over the path $\ell$ of the ray. The medium that is crossed by the ray is either the channel walls or the flow of particles we are interested in. Equation 1 can then be written:

$$
I(x, z)=I_{0} \exp \left[-\mu_{a t t}\left(\rho_{c w} 2 e+\rho_{P} \phi(x, z)\right)\right],
$$

where $e$ is the width of the chute sidewalls and $\rho_{c m}$ their density. The term $\phi$ is the volume fraction of the granular medium (inside the chute of width $W$ ) crossed by the X-ray that will reach the detector at point $(x, z)$. In order to get rid of the density of the empty channel, the calibration phase consists of measuring the intensity of the empty channel at every point $(x, z)$ of the detector $\hat{I}_{0}(x, z)=I_{0} \exp \left(\mu_{a t t} \rho_{c w} 2 e\right)$. For each point $(x, z)$ in the detector, the measured intensity is divided by the intensity of the the empty chute $\hat{I}_{0}$ to obtain:

$$
\frac{I(x, z)}{\hat{I}_{0}(x, z)}=\exp \left[-\mu_{a t t} \rho_{P} \phi(x, z) W\right] .
$$


The volume fraction of the granular medium at any location $(x, z)$ is then:

$$
\phi(x, z)=-c \ln \left(\frac{I(x, z)}{\hat{I}_{0}(x, z)}\right),
$$

where $c=1 /\left(\mu_{a t t} \rho_{P} W\right)$ is a constant. However, because the term $\mu_{\text {att }}$ in this constant remains an unknown, the coefficient $c$ needs to be determined experimentally. The volume fraction $\phi$ is a linear function of $-\ln \left(I / \hat{I}_{0}\right)$, meaning that the plot of $\phi$ versus $-\ln \left(I / \hat{I}_{0}\right)$ is a straight line which crosses zero with a slope $c$.

In order to get $c$, well defined configurations were considered when grains are static and the volume fraction is known. For those configurations, the X-ray intensity $\hat{I}_{f}$ of the channel full of static particles was measured at every point $(x, z)$ of the detector on the one hand, and the corresponding volume fraction was measured carefully as the mass of the particles times the density of one particle divided by the volume of the particles on the other hand. In the case of (spherical) glass beads, the random close packing configuration led to $\phi_{r c p}=0.62$. In the case of (elongated) rice grains, the calibration was done for two configurations: at the random close packing with a volume fraction found to be equal to 0.506 , and when the grains were oriented with a grain volume fraction found to be equal to 0.602 . This calibration process measuring both $\hat{I}_{0}$ and $\hat{I}_{f}$ allowed to establish the coefficient $c$ in Eq. 4 . Applying Eq. 4 to every point $(x, z)$ yields a complete widthaveraged field of volume fraction of the flow between the $\mathrm{X}$-ray source and the detector, where the jump is located, as it will be shown in the next section.

\section{Results}

\subsection{Examples of density fields across the granular jump}

Figure 3 shows two examples of spatial distribution of the volume fraction $\phi(x, z)$ across the granular jump measured from X-ray radiography, in the case of glass beads. Two rather extreme jump patterns are presented in order to highlight how the X-ray radiography is a robust and powerful tool to measure the flows of granular materials.

At high $Q_{m}$ and low $\zeta$ (top panel of Fig. 3), the incoming flow is thick and dense and it produces a very diffuse jump with gradual variation of the density field, and the flow after the jump is only slighly denser than the incoming flow. Interestingly, the X-ray technique allows to decipher the vertical (normal to the bottom) stratification of the density field (not discussed in the present paper): the panels of Fig. 3 suggest that the dense flows before and after the jumps are more dilute at the free-surface (yellow color) than into the core of the granular layer (red color).

At low $Q_{m}$ and high $\zeta$ (bottom panel of Fig. 3), the incoming flow becomes very thin and very dilute $(\phi<0.4)$ and it produce an extremely steep granular jump, and the flow after the jump is much thicker and denser. The transition between the incoming flow and the outgoing flow is far from being gradual and a sharp change in density is observed.
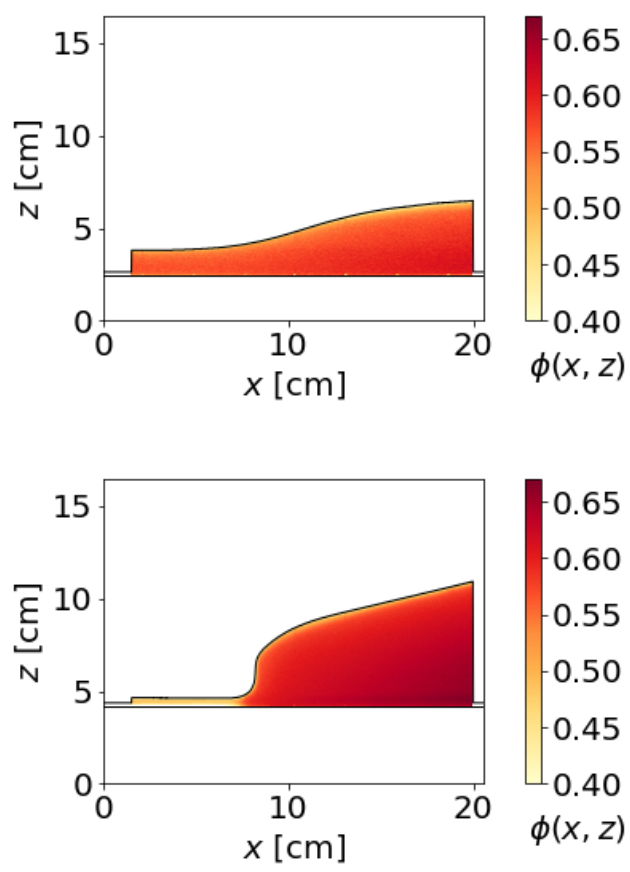

Figure 3. Examples of spatial distribution of the volume fraction $\phi(x, z)$ across the granular jumps, as measured by $\mathrm{X}$-ray radiography for two different input conditions (glass beads): $H / d=29.1$ and $\zeta=25^{\circ}$ (left panel) and $H / d=16.7$ and $\zeta=40^{\circ}$ (right panel). The values of volume fraction are by construction averaged over the chute width.

Interestingly, by comparing the flows after the jumps between the two panels of Fig. 3, it can be seen that the volume fraction reached at the bottom of the flows is consistently much larger when the thickness is increased.

\subsection{Free-surface and density profiles}

Figure 4 gives an example of a free-surface profile $(h(x))$ and the corresponding depth-averaged volume fraction profile $\phi(x)=\left(\int_{0}^{h} \phi(x, z) \mathrm{d} z\right) / h$ across a granular jump formed at $H / d=16.7$ and $\zeta=30^{\circ}$. In this case, the incoming flow is nearly uniform: it has a constant thickness before the jump, while its depth-averaged volume fraction is very slightly decreasing, thus showing (by mass conservation) that the flow is very slightly accelerating. The flow after the jump is non-uniform with a thickness that is gradually increasing and the corresponding volume fraction that is also increasing. This shows that the flow after the jump is (by mass conservation) strongly decelerating The transition from the nearly uniform, thin and fast, incoming flow towards that strongly decelerating, thick and slow, outgoing flow takes place through the granular jump. The granular jump takes place over a finite length along which a much stronger variation of the volume fraction occurs. The example shown on Fig. 4 shows that the Xray radiography is a robust and efficient technique to measure accurately the density variations that occur in granular flows, in particular across the jump which is accompanied 
by strong density gradients along the chute. The assumption of incompressibility often made in depth-averaged equations for the description of granular flows is reasonable before and after the jumps where the density gradients are nearly negligible (before the jump) or small (after the jump). However, that incompressibility assumption clearly fails for the granular flow throughout the jump.

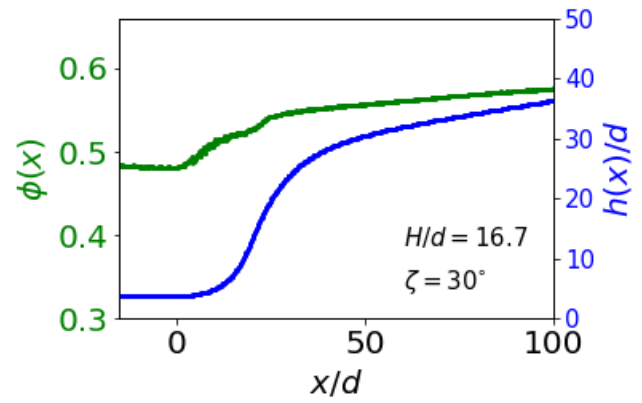

Figure 4. Free-surface (blue color) and depth-averaged volume fraction (green color) profiles along the chute in the vicinity of the granular jump, measured by the X-ray technique: example for $H / d=16.7$ and $\zeta=30^{\circ}$ (glass beads). The position $x=0$ corresponds to the beginning of the jump.

\section{Discussion and conclusion}

In the present paper, the use of X-ray radiography as an efficient tool to measure the density of granular flows along a chute was demonstrated under different flow states, in the nearly uniform flows before the jump and the graduallyvaried flows after the jump, as well as in the highly nonuniform flows across the jump. The frequency of the X-ray source $(30 \mathrm{~Hz})$ was not sufficient to get direct measurements of velocities but in the granular jump set-up as used in the present study, the depth-averaged velocity can be deduced from accurate height and volume fraction measurements by mass conservation because the flows are steady (mass discharge fully controlled by the opening height at the tank exit). Further developments are needed for direct velocity measurements of rapid granular chute-flows. It should be stressed that the values for height and volume fraction (and deduced velocity) are averaged values over the chute width. This is a crucial advantage of the X-ray radiography because the use of cameras at the sidewalls always gives results that are more difficult to interpret because of shearing of the flowing granular medium across the chute width (see for instance [2]). This short paper was dedicated to the description of the granular jump set-up and the use of X-ray radiography to measure the granular jumps, by showing some examples of consistent results in terms of free-surface and density profiles along the chute. A great number of experiments using X-ray radiography were done for different input mass discharges and slope angles of the chute. This allowed to obtain key dynamic features of the granular flows and the jumps formed during those flows over a relatively wide range of Froude numbers $(\mathcal{F}=\bar{u} / \sqrt{g h \cos \zeta})$ of the incoming flows. The full results are discussed in a detailed paper in preparation.

\section{Acknowledgments}

The authors are thankful to the Labex Tec21, which is part of the ANR "Investissements d'avenir" program for funding. S.M. is grateful to the funds provided by both the Idex Graduate School of University of Grenoble Alpes (ANR “Investissements d'avenir" program) and the ExploRa Doc scholarship from the Region AuvergneRhone-Alpes, France. T.F. thanks additional funding by French ministries "Ministère de l'Europe et des Affaires Etrangères" and "Ministère de l'Enseignement Supérieur, de la Recherche et de l'Innovation" through the PHC project GrainFlow within the FASIC 2017 program.

\section{References}

[1] A. Albaba, S. Lambert, T. Faug, Phys. Rev. E 97, 052903 (2018)

[2] T. Faug, P. Childs, E. Wyburn, I. Einav, Phys. Fluids 27, 073304 (2015)

[3] T. Faug, Can. Geotech. J. 58, 114-124 (2021)

[4] F. Guillard, B. Marks, I. Einav, Sci. Rep. 7, 8155 (2017)

[5] A.J. Jaworski, T. Dyakowski, Exp. Therm. Fluid Sci. 31, 877-885 (2007)

[6] A. Samadani, L. Mahadevan, A. Kudrolli, J. Fluid Mech. 452, 293-301 (2002)

[7] G. Smith, P. Rowley, R. Williams, G. Giordano, M. Trolese, A. Silleni, D.R. Parsons, S. Capon, Nat. Commun. 11, 2873 (2020)

[8] H. Xiao, J. Hruska, J.M. Ottino, R.M. Lueptow, P.B. Umbanhowar, Phys. Rev. E 98, 032906 (2018) 